\title{
Dipolar DC Collisional Activation in a "Stretched" 3-D Ion Trap: The Effect of Higher Order Fields on rf-Heating
}

\author{
Boone M. Prentice, Scott A. McLuckey \\ Department of Chemistry, Purdue University, 560 Oval Drive, West Lafayette, IN 47907-2084, USA
}

\begin{abstract}
Applying dipolar DC (DDC) to the end-cap electrodes of a 3-D ion trap operated with a bath gas at roughly 1 mTorr gives rise to 'ff-heating' and can result in collision-induced dissociation (CID). This approach to ion trap CID differs from the conventional single-frequency resonance excitation approach in that it does not rely on tuning a supplementary frequency to coincide with the fundamental secular frequeny of the precursor ion of interest. Simulations using the program ITSIM 5.0 indicate that application of DDC physically displaces ions solely in the axial (inter end-cap) dimension whereupon ion acceleration occurs via power absorption from the drive if. Experimental data shows that the degree of if-heating in a stretched 3-D ion trap is not dependent solely on the ratio of the dipolar DC voltage/radio frequency (rf) amplitude, as a model based on a pure quadrupole field suggests. Rather, ion temperatures are shown to increase as the absolute values of the dipolar DC and if amplitude both decrease. Simulations indicate that the presence of higher order multi-pole fields underlies this unexpected behavior. These findings have important implications for the use of DDC as a broad-band activation approach in multi-pole traps.
\end{abstract}

Key words: Dipolar DC collisional activation, Quadrupole ion trap, Stretched ion trap, Ion trap collision-induced dissociation

\section{Introduction}

$\mathrm{C}$ ollision induced dissociation (CID) is widely used in tandem mass spectrometry for the structural characterization of polyatomic gas-phase ions [1]. The fragmentation patterns noted in CID spectra are functions of the energetic and entropic requirements of the competing and consecutive decomposition channels, the time window of observation, and the internal energy distribution of the fragmenting ion population [2]. CID in contemporary tandem mass spectrometers can be effected over a wide range of conditions, including collision energy, numbers of collisions, and timescales. The accessible range of conditions depends upon the nature of the instrumentation, with ion traps and tandem time-of-flight instruments representing the two extremes.

Correspondence to: Scott A. McLuckey; e-mail: mcluckey@purdue.edu
Tandem time-of-flight instruments typically employ kiloelectronvolt laboratory frame collision energies with the likelihood for relatively few collisions in the collision region and time-scales on the order of microseconds. Ion trap CID, on the other hand, generally involves tens to hundreds of low electronvolt energy collisions over time frames of a millisecond to hundreds of milliseconds [3, 4]. Ion traps are popular tools in part due to the ability to conduct multiple mass selection steps with intervening reactions and are often used as stand-alone instruments or as part of a hybrid tandem mass spectrometer [5].

Ion trap CID has generally been induced through the application of a supplemental AC signal in resonance with the secular frequency of the ion to be activated, a technique often termed 'resonance excitation' [6, 7]. The AC signal acts to increase the amplitude of the ion's harmonic oscillation in the trap, thereby increasing its maximum displacement from the trap's center. As this displacement 
occurs, the ions visit areas of higher rf field where they can absorb power from the drive rf. Ion kinetic energies are increased in this overall process both as a result of the increase in velocity associated with the secular motion as well as the increase in velocities associated with power absorption from the drive rf, sometimes referred to as 'rfheating' [8]. Collisions with the bath gas at the elevated velocities associated with resonance excitation serve as means for conversion of kinetic energy to internal energy, thereby resulting in heating of the ions to the point where they may undergo fragmentation.

A number of alternative methods for accelerating ions in ion traps for the purpose of CID have been reported. Among these is 'boundary activated dissociation,' where the use of long DC pulses applied to the ring electrode of a 3-D ion trap positions ions close to the stability boundary and induces rf-heating [9-11]. However, as this method utilizes a quadrupolar DC potential, it induces a non-zero aparameter and alters the ion secular frequency [12]. By virtue of placing precursor ions very close to a stability boundary, it is not possible to capture product ions higher in mass-to-charge ratio than that of the precursor ion while also capturing product ions of lower mass-to-charge ratio. Such a capability is desirable, for example, for multiply charged precursor ions. Nonresonant ion acceleration has been described using low frequency $\mathrm{AC}$ waveforms applied to the end-cap electrodes [13]. CID associated with this form of ion acceleration has been attributed to energetic collisions associated with the restoring oscillatory motion response of trapped ions to the change in dipolar direction of the applied AC. Microsecond time-scale DC pulses have been applied either in a quadrupolar fashion to the ring electrode or in a dipolar fashion to the end-caps for the purposes of CID [14], surface-induced dissociation [15], phase-locking [16], and DC tomography experiments [17]. Plass has characterized the effects of the application of dipolar DC on ion motion, with particular emphasis on ion tomography studies, via simulations [18]. Perhaps most relevant to the work reported here is the description by Tolmachev et al. of the use of relatively long dipolar DC pulses (i.e., 1-100 ms) in a linear ion trap coupled with a Fourier-transform ion cyclotron resonance mass spectrometer for effecting CID [19]. The approach was coined dipolar DC collision-induced dissociation (DDC CID) and is based on the displacement of ions from the ion trap center, where rf fields are smallest, to regions of higher rf field strengths. Activation at these offcenter positions is due to the exposure of the ions to higher rf quadrupolar fields where increased power absorption from the main rf trapping voltage occurs, as reflected by an increase in the so-called 'micro-motion' or rf-ripple (see Figure 1 for a schematic depiction of the phenomenon). A convenient way to follow changes in the extent of ion activation in the ion trap is via measurement of the rate at which precursor ions are converted to product ions. Tolmachev et al. presented a model that relates dipolar DC operating conditions to the effective internal temperature to which the precursor ion can be elevated [19]. We have recently described the implementation of dipolar DC in a quadrupole collision cell of a quadrupole/time-of-flight tandem mass spectrometer [20]. We have also recently reported CID methods in a 3-D ion trap which utilize a monopolar DC field [21] and a dipolar DC field [22]. DDC CID is believed to be mechanistically very similar using monopolar or dipolar DC; however, the monopolar DC variant leads to a significant change in the value of the aparameter and is thus more prone to discrimination against ions of high mass-to-charge ratio. In some respects, DDC CID is expected to be very similar to conventional resonance excitation in that both are "slow heating" methods [4]. However, in some respects, the two approaches to ion acceleration are complementary. For example, unlike resonance excitation, the DC method lacks a resonance condition in that all ions are displaced from the ion trap center regardless of mass-to-charge ratio. In this respect, the DC method is a 'broad-band' technique. As a consequence, unlike with resonance excitation where first generation product ions are collisionally cooled, DDC CID first generation product ions are also exposed to rf-heating such that consecutive dissociation reactions are more likely. In this regard, DDC CID has analogies with ion trap photodissociation (PD) techniques [23]. This report describes simulations, using the ion trap simulation program ITSIM 5.0 [24], and experiments intended to shed light on underlying phenomena that affect DDC CID in a 3-D quadrupole ion trap with intentionally introduced higher order multi-pole fields.

\section{Experimental}

\section{Instrumentation}

All experiments were performed on a dual source Finnigan ion trap mass spectrometer (ITMS, ThermoFinnigan Corp., San Jose, CA, USA), modified to allow for ion/ion reactions as previously described [25]. Briefly, ions from one of two sources can be sequentially injected axially into the ion trap by means of a DC turning quadrupole. The injection and timing are controlled using Ion Catcher Mass Spectrometer software (ICMS Ion Trap Software ver. 2.20, University of Florida). Application of the DDC pulse was performed as described elsewhere [20]. Briefly, the pulse was initiated from an Agilent 8114A Programmable Pulse Generator (Agilent Technologies, Santa Clara, CA, USA) by a transistor-transistor logic (TTL) trigger in the software. The DC pulse from the pulse generator was run through home built APEX amplifiers (Cirrus Logic, Inc., Austin, TX, USA), powered by two Kikisui PMC350-0.2A power supplies (Kikisui America, Inc., Burlingame, CA, USA) and a three output Agilent E3630A power supply (Agilent Technologies, Santa Clara, CA, USA), which provided two amplified outputs opposite in polarity and equal in magnitude. The outputs from the amplifier ran into a custom built 


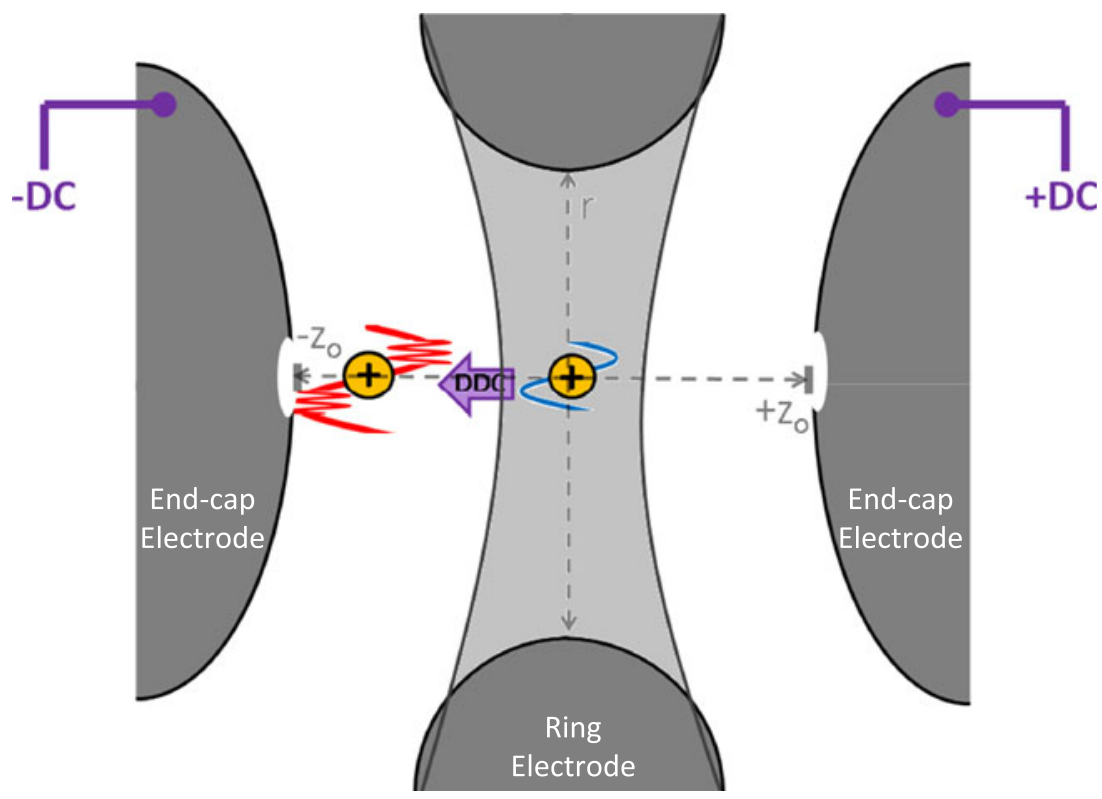

Figure 1. Schematic depiction of the dipolar DC (DDC) experiment with the 3-D ion trap wherein the main rf voltage is applied to the ring-electrode. DDC acts to physically displace the ion population from the center of the trap in the z- (or inter end-cap) dimension where the ions experience larger if electric fields. The increase in the ion velocity distribution is largely due to enhanced micro-motion or ripple, which is represented here as the oscillatory motion on the red sine-wave that represents the ion motion after application of the DDC

switchbox, which was toggled by the TTL trigger in the software, as mentioned, between the DC pulse channels and the channels for supplemental dipolar AC as provided from the Finnigan electronics. Two resistors in series on each output, an $820 \mathrm{k} \Omega$ and $890 \mathrm{k} \Omega$ (totaling $1710 \mathrm{k} \Omega$ ), were placed between the outputs of the APEX amplifier and the switchbox in order to prevent the drive $\mathrm{rf}$ (which is capacitively coupled to the end-cap electrodes) from sinking back to the amplifying circuitry and distorting the pulse as well as the rf field within the trap.

A second pulse configuration, which uses custom-built band reject PI-based notch filters in order to eliminate the shunting of the drive rf back to the amplifying circuitry, has also been utilized as described previously [20]. Briefly, these filters are placed in series between each output of the APEX amplifier and the trap, thereby eliminating the need for the protecting resistors and the time sharing functions provided by the switchbox. This setup modulates the supplemental AC signal through the APEX circuitry unamplified and applies it to the end-caps in a dipolar fashion, allowing for the application of simultaneous AC and DDC waveforms.

\section{Materials}

Leucine enkephalin (YGGFL) was purchased from Sigma Aldrich (St. Louis, MO, USA) and methanol was purchased from Mallinckrodt (Phillipsburg, NJ, USA). Stock solutions of $1.0 \mathrm{mg} / \mathrm{mL}$ were prepared in 50:50 methanol/water solutions and then diluted up to 3 -fold prior to use in nanoelectrospray ionization (nESI).

\section{Simulations}

Simulations were performed using the ion trap simulation program ITSIM ver. 5.0 [24]. Simulations were conducted using the Finnigan ITMS stretched ion trap geometery [26] at an $\mathrm{rf}$ drive frequency of $1.1 \mathrm{MHz}$ in order to mimic experimental trap conditions. Fourth order Runge-Kutta integration was performed using $10 \mathrm{~ns}$ integration steps. Langevin collision model was used at a bath pressure of 1 mTorr (helium, $\mathrm{T}=300 \mathrm{~K}, \mathrm{M}=4.0026 \mathrm{u}$, polarizability= $0.205 \AA^{3}$ ), unless otherwise noted. Ions over a range of mass-to-charge values were simulated using $50 \AA^{2}$ cross sections with internal energy transfer and fragmentation disabled, unless otherwise noted. Typically, the DDC voltage was applied after an initial cooling period $(\sim 0.5 \mathrm{~ms})$ from the start of the simulation. Typically, the $\mathrm{V}_{\mathrm{DDC}}$ was ramped to its final voltage, both upon initiation and upon cessation, over $50 \mu \mathrm{s}$ in order to simulate the rise (to a final voltage of ' $x V_{\text {DDC }}$ ') and fall (to $0 V_{D D C}$ ) times of the DDC pulse in the experimental setup. Instantaneous application or removal of the pulse can result in trajectory displacements dependent on the phase of ion oscillation upon pulse initiation, as has been reported previously with DC tomography experiments [17]. ITMS stretched trap default multipole expansion coefficients were utilized unless otherwise noted. These include ring electrode quadrupole terms (dipole $=$ 0 , quadrupole $=0.8945$, hexapole $=0$, octopole $=0.0158$, decapole $=0$, dodecapole $=0.0076$ ), left end-cap monopole terms (dipole $=-0.4929$, quadrupole $=0.44725$, hexapole $=-0.2394$, octopole $=0.0079$, decapole $=0.0234$, dodecapole $=0.0038$ ), right end-cap monopole terms (dipole $=0.4929$, quadrupole $=$ 
0.44725 , hexapole $=0.2394$, octopole $=0.0079$, decapole $=-$ 0.0234 , dodecapole $=0.0038$ ), and $\mathrm{z}$ dipole terms (dipole $=$ 0.4929 , hexapole $=0.2394$, decapole $=-0.0234$ ). In discussions in which 'high order' terms are neglected, this refers to setting terms higher than quadrupole (i.e., hexapole, octopole, decapole, and dodecapole) for the left end-cap monopole, right end-cap monopole, and $\mathrm{z}$ dipole components equal to zero. Ring electrode quadrupole terms were left default in all simulations.

\section{Results and Discussion}

\section{Ion Trajectory Characterization}

DDC CID is effected by applying DC voltages to the endcap electrodes of the 3-D ion trap in a $+/$ - fashion (i.e., a "+y" DC voltage on one end-cap electrode and a "-y" DC voltage on the opposing end-cap electrode). Single ion simulations of $\mathrm{m} / \mathrm{z} 133$ ions (e.g., $\mathrm{Cs}^{+}$) were performed to characterize ion motion in the presence of the DDC field. The simulations indicate that application of the DDC field displaces ions in the axial (z) dimension while the radial ( $\mathrm{x}$ and $\mathrm{y}$ ) components of ion motion remain unperturbed (Figure 2a). Upon initiation of a pulse at $\mathrm{t}=0.5 \mathrm{~ms}$, the center of the ion's harmonic oscillation is displaced solely in the $\mathrm{z}$ direction by roughly $4 \mathrm{~mm}$, a magnitude dependent upon the ion $m / z$, the $\mathrm{V}_{\mathrm{rf}}$ trapping conditions (low mass cut-off (LMCO), which was $40 \mathrm{Th}$ in this case), and the magnitude of the DDC voltage $(40 \mathrm{~V}$, which corresponds to $+20 \mathrm{~V}$ DC on one end-cap and $-20 \mathrm{~V}$ DC on the opposing end-cap). Figure $2 \mathrm{~b}$ plots ion velocity in the three dimensions for the same simulation. The sensitivities of ion displacement and velocity to these parameters are discussed further below. Removal of the DDC field at $\mathrm{t}=1.55 \mathrm{~ms}$ allows the ion to undergo cooling collisions with the bath gas and relax back to the center of the ion trap.

Figure 2c shows an expansion of the results of Figure 2a and $b$ around the time of pulse initiation to clarify the mechanism that underlies the increase in ion velocity. Prior to DDC pulse initiation, the ion's oscillatory behavior is dominated by its secular motion with relatively small contribution from rf ripple, also referred to as micromotion. As the DDC pulse is applied, the ion is displaced from the center of the ion trap in the axial dimension. As this occurs, the ion is exposed to higher rf field strengths associated with the drive trapping rf potential (applied to the ring electrode). This is evident through the superposition of the $1.1 \mathrm{MHz}$ drive $r f$ frequency upon the secular oscillatory motion of the ion. Indeed, the major change in ion motion associated with the application of DDC is a dramatic increase in the contribution due to micromotion. While the center of the ion's axial motion is altered by the DDC field, the total oscillatory amplitude changes modestly. For example, the total oscillatory amplitude prior to DDC activation in the simulation of Figure 2 is from $0.6 \mathrm{~mm}$ to $-0.6 \mathrm{~mm}$, a total amplitude of roughly $1.2 \mathrm{~mm}$. During DDC activation, the oscillatory motion is from $-2.9 \mathrm{~mm}$ to $-4.8 \mathrm{~mm}$, a total amplitude of $1.9 \mathrm{~mm}$. The change is essentially entirely due to the increase in the amplitude of the micromotion with the amplitude of the secular motion being more-or-less constant.

Of direct relevance to CID is the change in ion velocity associated with application of DDC, which is shown in Figure $2 \mathrm{~b}$ and $\mathrm{c}$. As it is clear that the main change in ion oscillatory amplitude arises from the micromotion, it follows that it is the micromotion that contributes to the increase in ion velocity. Upon initiation of the pulse at $\mathrm{t}=0.5 \mathrm{~ms}$, the axial ion velocity is dramatically increased, despite the modest increase in total oscillatory amplitude mentioned previously. This is due to the greater relative contribution to ion velocity from the micromotion as a result of its higher frequency relative to that of the secular motion. The oscillatory amplitude prior to application of DDC and at relatively low q-values is largely due to secular motion. Micromotion in the absence of any DDC is, on the other hand, of comparatively small amplitude. Upon displacement from the trap center, the amplitude of the micromotion increases dramatically. The relative frequency contribution of the micromotion component compared with the secular motion component increases roughly 10 -fold in the presence of the DDC compared with no applied DDC, as analyzed by performing fast Fourier transforms (FFT) on the axial trajectories using ITSIM. It is the change in the oscillatory amplitude due to the micromotion that underlies the increase in velocity.

Similar to resonance excitation, DDC excitation is a slow heating method whereby the precursor ions reach an elevated steady state internal energy distribution after a transition period from the initial ion internal energy distribution [3]. Once this steady-state internal energy distribution is reached, pseudo-first-order dissociation kinetics are observed for a common population of precursor ions. Tolmachev and coworkers derived a model to relate experimental variables to the change in effective ion temperature in the DDC experiment, assuming purely quadrupolar and dipolar fields [19], as shown in Equation (1):

$$
\Delta T_{K}=\frac{m_{g} \Omega r_{o}^{2}}{24 k_{b}}\left(\frac{V_{D D C}}{V_{R F}}\right)^{2}
$$

where $\mathrm{m}_{\mathrm{g}}$ is the mass of the bath gas, $\Omega$ is the $\mathrm{rf}$ drive frequency, $r_{0}$ is the radius, $V_{r f}$ is the amplitude of the $r f$ drive voltage, $\mathrm{V}_{\mathrm{DDC}}$ is the amplitude of the dipolar DC, and $\mathrm{k}_{\mathrm{b}}$ is the Boltzmann constant [19]. From a qualitative standpoint, the dipolar DC voltage can be considered as the potential that moves the ion cloud from the ion trap center while the rf amplitude determines how strongly the ions are held at the center. Equation (1) suggests that the change in ion internal temperature is independent of the precursor ion mass-tocharge ratio and that the change in internal temperature should be constant at constant $\mathrm{V}_{\mathrm{DDC}} / \mathrm{V}_{\text {rf }}$ ratio. We have tested this prediction both by experiment and by simulation for the stretched 3-D ion trap. 
(a)

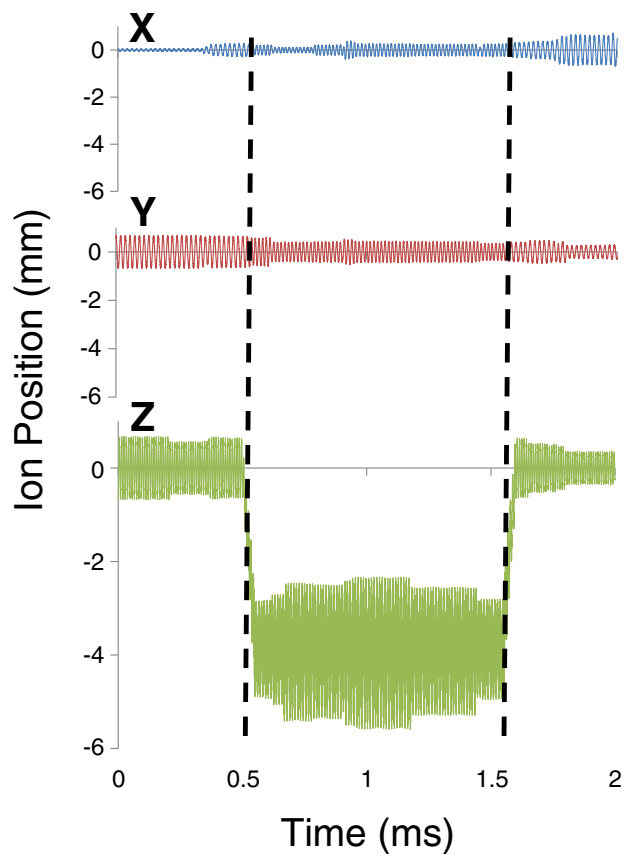

(b)

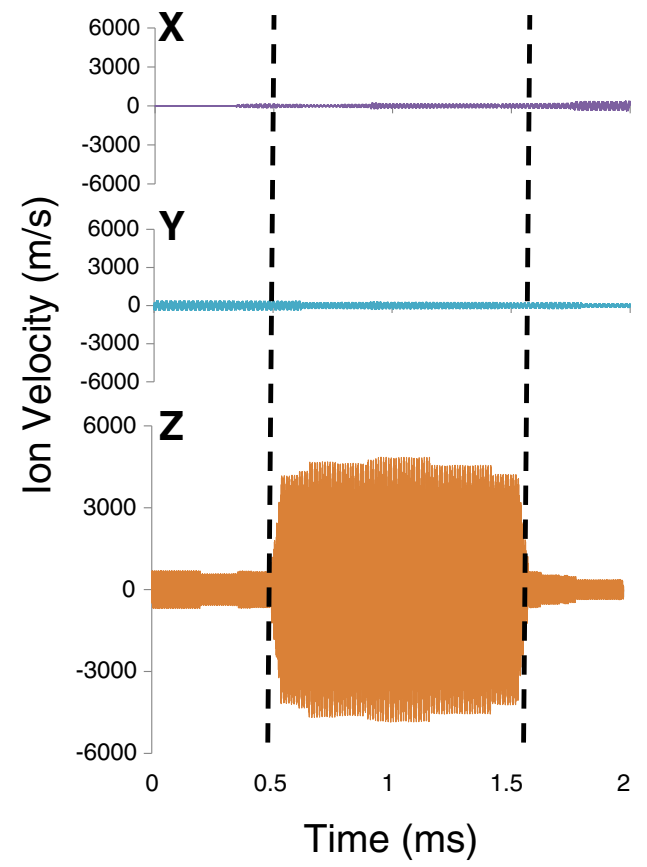

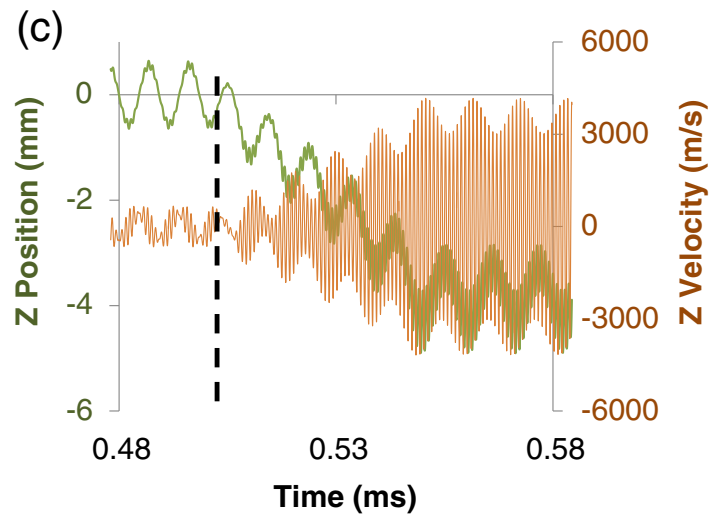

Figure 2. Single ion simulation of an ion of $\mathrm{m} / \mathrm{z} 133$ at a $L M C O=40 \mathrm{Th}(\mathrm{q}=0.27)$ in which the DDC pulse is initiated at $\mathrm{t}=0.5$ milliseconds and ramped linearly to $40 \mathrm{~V}$ in 50 microseconds. The pulse is removed in the same ramped fashion at $t=1.55$ miliseconds. (a) lon position as a function of time. (b) Ion velocity as a function of time. (c) Expansion of (a) and (b) over the period of $0.48-0.58$ milliseconds

Protonated leucine enkephalin is a commonly used thermometer ion [27] in mass spectrometry that has been examined over a wide range of conditions, including thermal dissociation [28], ion trap resonance excitation [29], and boundary activation [30]. The rate of precursor ion dissociation is a measure of the precursor ion internal energy distribution. Under appropriate conditions (i.e., the rapid energy exchange condition [31]), the measured dissociation rate is related to the internal energy distribution, which can be described by a temperature, via the Arrhenius equation. Master equation modeling of the dissociation of protonated leucine enkephalin under typical ion trap conditions suggests that this condition is met for dissociation rates less that $1 \mathrm{~s}^{-1}$ [3], which is significantly lower than dissociation rates normally induced in ion trap CID. Nevertheless, while the precursor ion internal energy distribution may not be Boltzmann, the dissociation rate is sensitive to differences in precursor ion internal energy. Therefore, similar dissociation rates should be observed at similar $\mathrm{V}_{\mathrm{DDC}} / \mathrm{V}_{\mathrm{rf}}$ ratios, according to Equation (1). Figure 3a shows kinetic data for protonated leucine enkephalin under conditions of minimal total ion loss using various combinations of $\mathrm{V}_{\text {DDC }}$ and $\mathrm{V}_{\text {rf }}$ that all gave a $\mathrm{V}_{\mathrm{DDC}} / \mathrm{V}_{\text {rf }}$ ratio of 0.04 . The observed loss rates are due to dissociation and they are clearly observed to vary with the absolute values of $\mathrm{V}_{\mathrm{DDC}}$

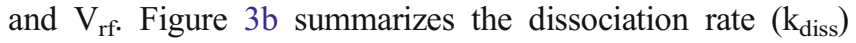
results as a function of the $\mathrm{V}_{\mathrm{DDC}}$ used to collect the data. The results show a monotonic decrease in dissociation rate as the magnitudes of both the DDC and rf voltages are increased at a constant ratio. 
(a)

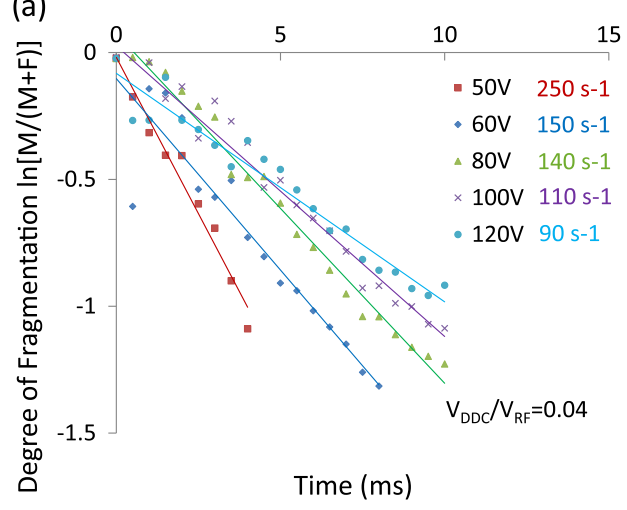

(b)

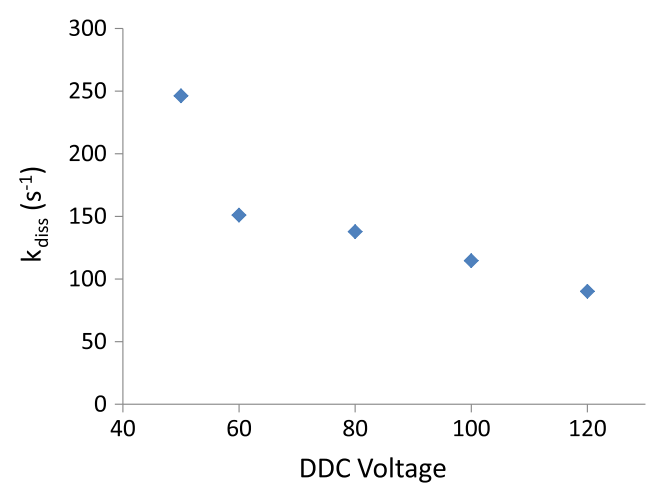

Figure 3. (a) Experimentally determined dissociation rates of leucine enkephalin at various magnitudes of $V_{D D C}$ (as indicated in the legend) and $V_{\text {rf }}$ but at a constant $V_{D D C} N_{\text {rf }}$ ratio of $\sim 0.04$ and (b) experimental dissociation rates under the specified activation conditions of leucine enkephalin as a function of applied DDC voltage

The kinetic data of Figure 3 suggest that the internal energy deposition into the leucine enkephalin ions is inversely related in some way to the absolute values of $\mathrm{V}_{\mathrm{DDC}}$ and $\mathrm{V}_{\mathrm{rf}}$ at a constant $\mathrm{V}_{\mathrm{DDC}} / \mathrm{V}_{\mathrm{rf}}$ ratio, which is not anticipated by Equation (1). This led to the hypothesis that higher order multipole fields, which are known to be present in the Finnigan stretched ion trap geometry, give rise to this behavior. Figure 4 shows the effect of higher order multipole fields on ion displacement and ion velocity based on simulation. In the presence of all default multipole coefficient terms for an ITMS stretched trap geometry (i.e., dipole, quadrupole, hexapole, octapole, decapole, and dodecapole fields), the simulated axial ion displacement for an ion arbitrarily chosen to be of $\mathrm{m} / \mathrm{z} 175$ in the presence of 30 $\mathrm{V}_{\text {DDC }}$ at a LMCO of $40 \mathrm{Th}$ is roughly $4.5 \mathrm{~mm}$. When the high order multipole expansion coefficients for the end-cap (a)

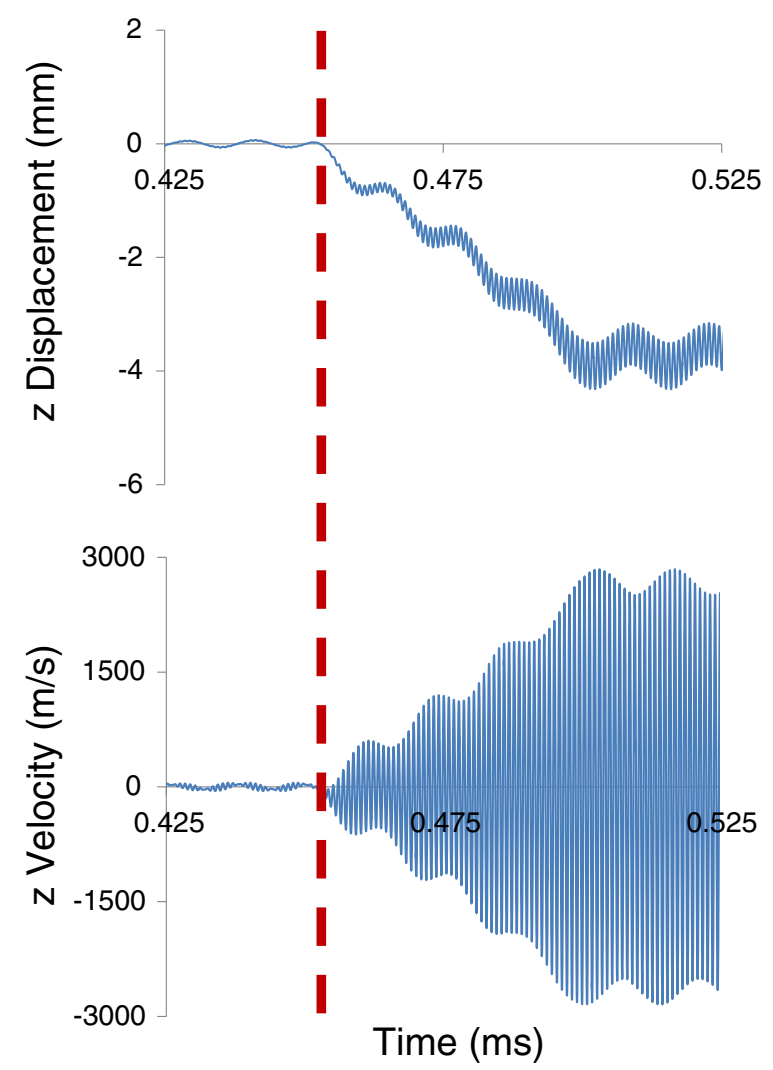

(b)

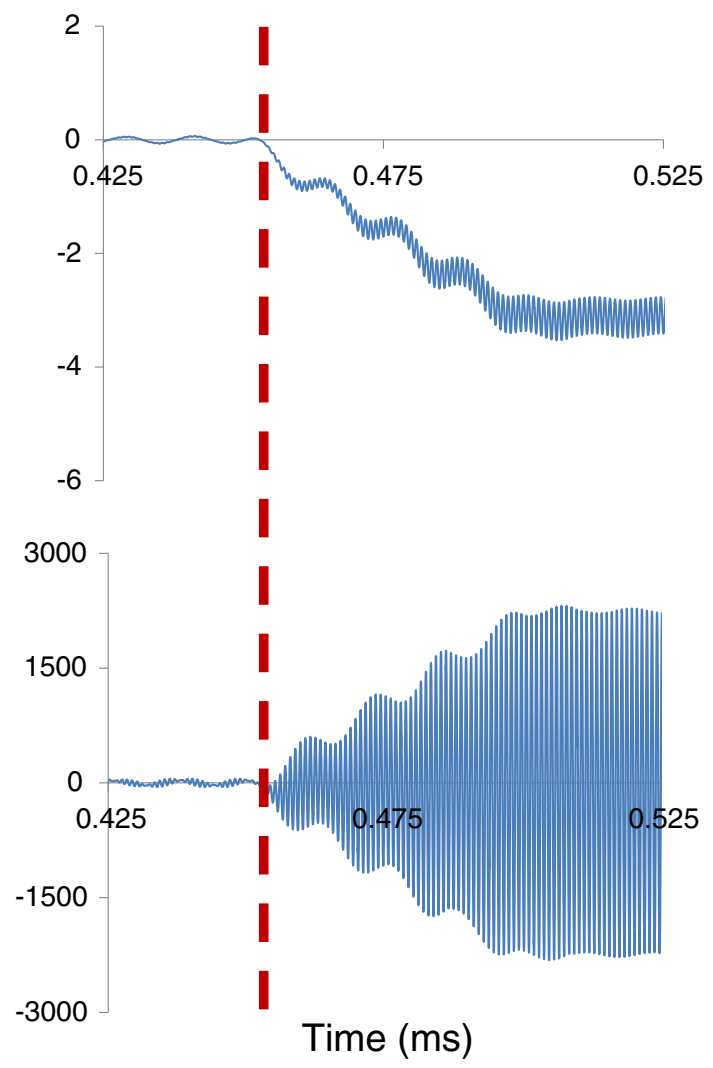

Figure 4. Axial displacement and axial velocity of an $m / z 175$ ion under $30 \mathrm{~V}_{\mathrm{DDC}}$ at a LMCO of $40 \mathrm{Th}$ (a) in the presence of typical ITMS stretched trapping fields which include dipole, quadrupole, hexapole, octapole, decapole, and dodecapole expansion coefficients and (b) in the presence of only dipole and quadrupole ITMS stretched trap expansion coefficient field terms 
monopoles (i.e., hexapole, octapole, decapole, and dodecapole) and, more significantly, the axial dipole (i.e., hexapole and decapole) were set to zero, the same simulated conditions resulted in an axial displacement of only roughly $3.5 \mathrm{~mm}$ (note that the term 'high order' refers to any term greater than the quadrupolar term). In this instance, the ring electrode quadrupole expansion coeffcients (i.e., dipole, quadrupole, hexapole, octapole, decapole, and dodecapole) are left default. The resulting ion velocity profile also clearly showed higher maximum velocities when the higher order components were present.

The simulations of Figure 4 indicate that the higher order fields of the stretched ion trap can affect both ion displacement and ion velocity. To determine if the higher order fields can also underlie the observed dependence of dissociation rate on the absolute values of $\mathrm{V}_{\mathrm{DDC}}$ and $\mathrm{V}_{\mathrm{rf}}$ at constant $\mathrm{V}_{\mathrm{DDC}} / \mathrm{V}_{\mathrm{rf}}$ ratio, a series of simulations were conducted with a range of precursor ion masses. The ion velocites were converted to effective temperatures, $T_{\text {eff, }}$ using the relationship [19]:

$$
T_{\text {eff }}=\frac{m_{r}}{m_{g}} T+\frac{m_{r}\left\langle v_{i}^{2}\right\rangle}{3 k_{b}}
$$

where $\mathrm{m}_{\mathrm{r}}$ is the reduced mass and $<\mathrm{v}_{\mathrm{i}}^{2}>$ is the mean squared velocity. The plots of Figure $5 \mathrm{a}$ and $\mathrm{b}$ compare ion temperatures (diamonds) calculated from ion velocities, and maximum displacements (squares) from simulations of ions over a range of mass-to-charge values with (Figure 5a) and without (Figure 5b) the inclusion of contributions from higher order fields in the ion trap. In all cases, the maximum displacement was greater with the inclusion of the higher order fields and the difference between displacement with and without higher order fields was greater as the ion massto-charge increased. Likewise, ion temperatures were greater in the presence of higher order fields and the difference

(a)

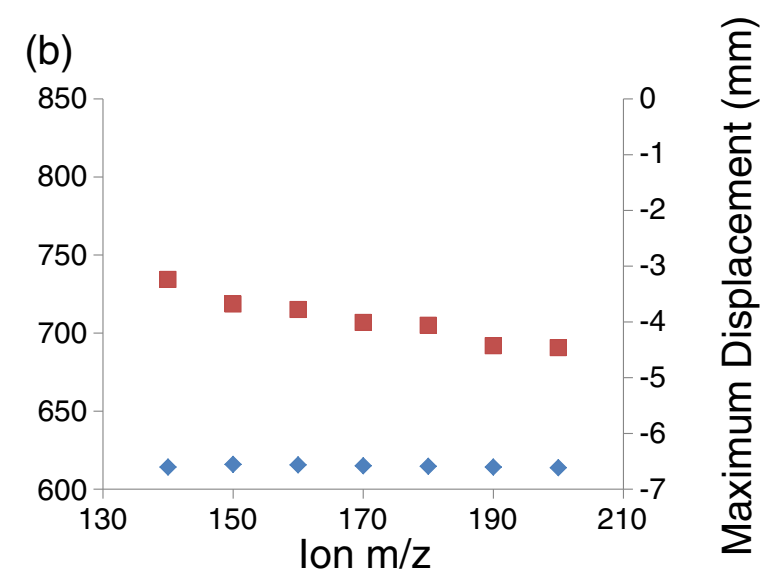

(c)
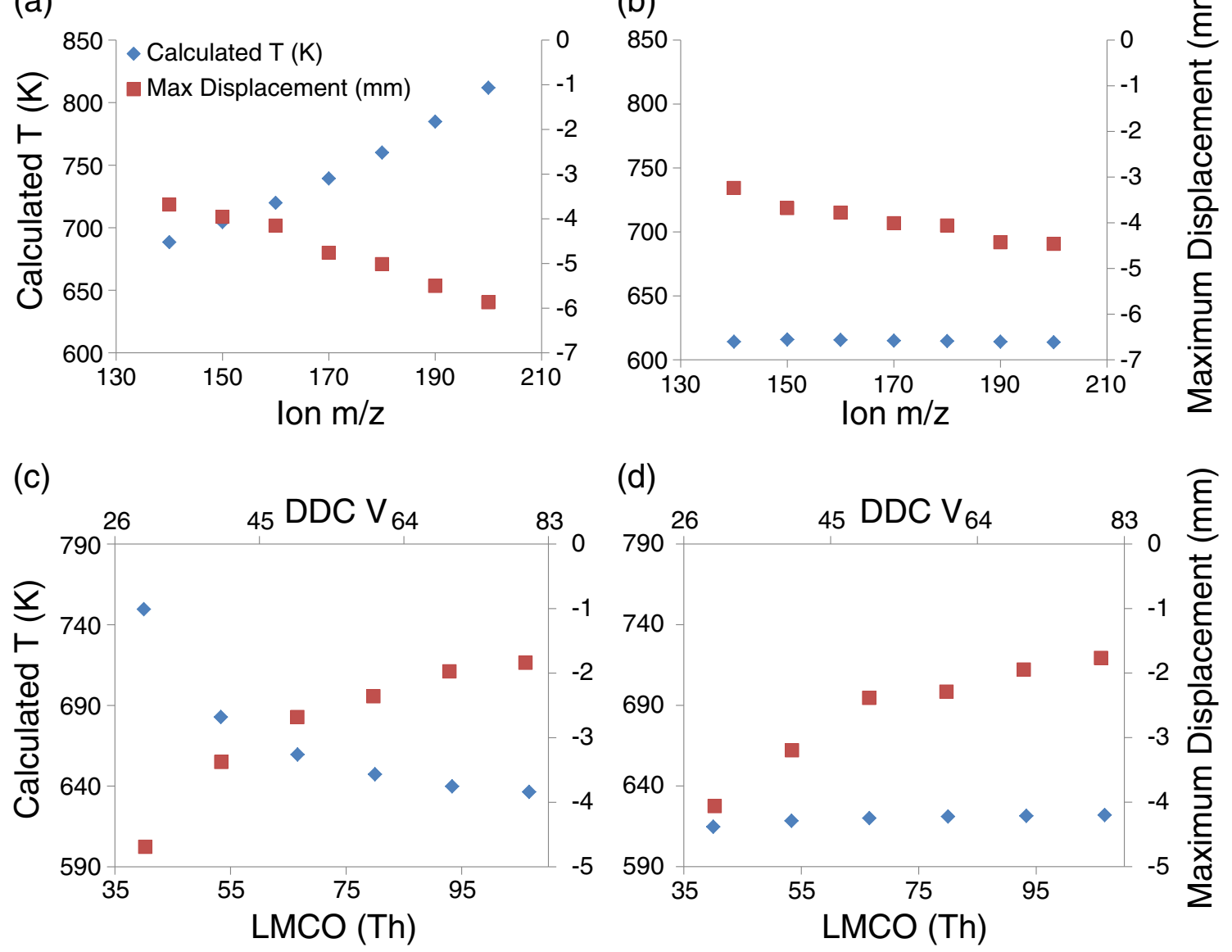

Figure 5. Simulations in an ITMS stretched trap showing ion displacement (squares) and effective ion temperature (diamonds), as calculated using ion velocities per Equation (2), as a function of ion $\mathrm{m} / z$ using $\mathrm{V}_{\mathrm{DDC}}=30 \mathrm{~V}$ at a LMCO of 40 Th (a) in the presence of all ITSIM default high order field components and (b) in the presence of only the dipole and quadrupole expansion coefficient terms. Simulations in an ITMS stretched trap showing ion displacement and effective ion temperature for $175 \mathrm{~m} / \mathrm{z}$ ions activated at various magnitudes of $V_{D D C}$ and $V_{\text {rf }}$, but at a constant $V_{D D C} N_{\text {rf }}$ ratio of $\sim 0.06$ (c) in the presence of default high order fields and (d) in the presence of only dipole and quadrupole field terms 
increased with mass-to-charge ratio. As predicted by Equation (1), the final steady state temperature of the ion was found to be independent of the mass-to-charge ratio of the ion when no higher order fields were included in the simulation. These results are particularly significant in that they show that the final steady state effective ion temperatures of the ions are $m / z$-dependent when higher order field components are present in the ion trap, which is not predicted in the model that assumes quadrupolar and dipolar fields only.

Figure $5 \mathrm{c}$ and $\mathrm{d}$ compare plots of ion temperatures (diamonds) and maximum displacements (squares) derived from simulations with (Figure 5c) and without (Figure 5d) higher order fields for ions of $m / z 175$ at various $V_{\mathrm{DDC}}$ and $\mathrm{V}_{\mathrm{rf}}$ values (the latter represented as LMCO in the figure) but with the same $V_{\mathrm{DDC}} / \mathrm{V}_{\mathrm{rf}}$ ratio. As predicted by Equation (1), the ion temperature was found to be essentially independent of the absolute values of $\mathrm{V}_{\mathrm{DDC}}$ and $\mathrm{V}_{\mathrm{rf}}$ (i.e., invariant with constant $\mathrm{V}_{\mathrm{DDC}} / \mathrm{V}_{\mathrm{rf}}$ ) when no higher order fields are included whereas the ion temperature was strongly dependent upon the values of $V_{D D C}$ and $V_{r f}$ when the higher order fields were included in the simulation. In all cases, the maximum displacement increases as the pseudo-potential well-depth decreases. In the case of the plots of Figure 5a and b, well-depth decreases as the mass-tocharge ratio of the ion increases whereas in Figure $5 \mathrm{c}$ and d, well-depth decreases as the LMCO decreases. The maximum displacement is larger in the presence of higher order fields than in the absence of higher order fields in all cases but the difference in maximum displacements increases as well-depth decreases. The increase in rf-heating noted here due to the presence of higher change multi-pole fields may very well underlie the so-called multi-pole storage assisted dissociation phenomenon noted in hexapole ion guides/traps [32]. Under high ion density conditions, CID that is highly analogous to ion trap CID has been noted. It has been proposed that ion-ion repulsion can force ions into regions of electric field that results in larger amplitude secular oscillations [33]. An alternative interpretation is that the mutual repulsion of the ions serves a role analogous to DDC by moving the ions into regions of relatively high multi-pole electric field strengths where they can undergo rf-heating that results in CID. Both phenomena may occur but the increased rf-heating due to the hexapole field is likely the stronger contributor to CID. It is noteworthy that the dramatic effects observed in hexapoles at high ion densities has not been noted with quadrupole devices, which is consistent with the higher degree of rf-heating noted here when higher order fields are present. The results noted here for the 3-D quadrupole ion trap with significant higher order field components are relevant to the use of DDC in that the maximum ion displacements and ion temperatures are higher than for ion traps with purely quadrupolar fields. Unlike purely quadrupole ion traps, the ion temperatures are dependent upon the $m / z$ value of the ion. Furthermore, since maximum ion displacements are higher in the presence of higher order fields, the range of $\mathrm{m} / \mathrm{z}$ ions that can be subjected to DDC is narrower because high $\mathrm{m} / \mathrm{z}$ ions undergo ion evaporation at lower DDC values when the higher order fields are present.

\section{Conclusions}

DDC activation in a 3-D ion trap, whereby equal and opposite DC voltages are applied to the end-cap electrodes, has been shown through simulations to displace and accelerate ions solely in the axial direction. Ion acceleration manifests itself largely in the form of ion micro-motion induced by the drive rf potential (i.e., rf-heating) as ions are displaced further from the center of the trap. Unlike resonance excitation, in which first generation product ions are collisionally cooled, DDC activation represents a continual heating process where product ions may also undergo rf-heating. However, the presence of significant nonlinear higher order multi-pole components, as is the case with the "stretched ion trap," introduces nonuniformity in the extent of rf-heating for ions of different $\mathrm{m} / \mathrm{z}$ under a fixed set of DDC conditions. This stands in contrast to ion traps with purely quadrupolar fields. In the latter case, DDC ion heating at a fixed drive frequency, ion trap radius, and background gas is dependent only upon the $\mathrm{V}_{\mathrm{DDC}} / \mathrm{V}_{\mathrm{rf}}$ ratio. The presence of higher order fields gives rise to less heating for product ions of lower mass-to-charge ratio and greater heating for product ions of higher mass-to-charge ratio because the higher $\mathrm{m} / \mathrm{z}$ ions are displaced further from the center of the ion trap, thereby exposing them to higher electric field strengths associated with the drive rf . At extreme displacements, it is important to note that ion evaporation can compete with dissociation, resulting in discrimination of high $\mathrm{m} / \mathrm{z}$ ions under certain operating conditions. The maximum ion displacement is also higher in the stretched ion trap than in the pure quadrupolar ion trap under a given set of DDC conditions. The difference in maximum ion displacement increases as the pseudo-potential well depth of the trapped ions decreases, which suggests that the range of ions that can be stored simultaneously is lower when higher order fields are present due to greater discrimination against high $\mathrm{m} / \mathrm{z}$ ions. These results suggest that the judicious incorporation of higher order fields might be considered in multi-pole storage devices that employ DDC as an activation method.

\section{Acknowledgments}

The authors acknowledge Jim Zimmerman, Robert Oglesbee, and Robert Santini of the Jonathan Amy Facility for Chemical Instrumentation for their support. This work was sponsored by the Office of Basic Energy Sciences, Office of Science, U.S. Department of Energy, under Award DE-FG0200ER15105.

\section{References}

1. Wells, J.M., McLuckey, S.A.: Collision-induced dissociation (CID) of peptides and proteins. Methods Enzymol. 402, 148-185 (2005)

2. McLuckey, S.A.: Principles of collisional activation in analytical mass spectrometry. J. Am. Soc. Mass Spectrom. 3, 599-614 (1992)

3. Goeringer, D.E., McLuckey, S.A.: Evolution of ion internal energy during collisional excitation in the Paul Ion Trap: A stochastic approach. J. Chem. Phys. 104, 2214-2221 (1996) 
4. McLuckey, S.A., Goeringer, D.E.: Slow heating methods in tandem mass spectrometry. J. Mass Spectrom. 32, 461 (1997)

5. McLuckey, S.A., Wells, J.M.: Mass analysis at the advent of the $21^{\text {st }}$ century. Chem. Rev. 101, 571-606 (2001)

6. Louris, J.N., Cooks, R.G., Syka, J.E.P., Kelley, P.E., Stafford Jr., G.C., Todd, J.F.J.: Instrumentation, applications, and energy deposition in quadrupole ion-trap tandem mass-spectrometry. Anal. Chem. 59, 16771685 (1987)

7. Wang, M., George III, J.E.: Technology Progress and Application in GC/MS and GC/MS/MS. In: March, R.E., Todd, J.F.J. (eds.) Practical Aspects of Trapped Ion Mass Spectrometry, vol. V. CRC Press, Boca Raton, FL (2009). Chapter 15

8. Goeringer, D.E., Viehland, L.A., Danailov, D.M.: Prediction of collective characteristics for ion ensembles in quadrupole ion traps without trajectory simulations. J. Am. Soc. Mass Spectrom. 17, 889-902 (2006)

9. Paradisi, C., Todd, J.F.J., Vettori, U.: Boundary effects and collisional activation in a quadrupole ion trap. Org Mass Spectrom 27, 251-254 (1992)

10. Traldi, P., Catinella, S., March, R.E., Creaser, C.S.: Boundary Excitation. In: March, R.E., Todd, J.F.J. (eds.) Practical Aspects of Ion Trap Mass Spectrometry, vol. I. CRC Press, Boca Raton, FL (1995). Chapter 7

11. Vachet, R.W., Glish, G.L.: Boundary-activated dissociation of peptide ions in a quadrupole ion trap. Anal. Chem. 70, 340-346 (1998)

12. Splendore, M., Lausevic, M., Lausevic, Z., March, R.E.: Resonant excitation and/or ejection of ions subjected to DC and rf fields in a commercial quadrupole ion trap. Rapid Commun. Mass Spectrom. 11, 228-233 (1997)

13. Wang, M., Schachterle, S., Wells, G.: Application of non-resonance excitation to ion trap tandem mass spectrometry and selected ejection chemical ionization. J. Am. Soc. Mass Spectrom. 7, 668-676 (1996)

14. Lammert, S.A., Cooks, R.G.: Pulsed axial activation in the ion trap: A new method for performing tandem mass spectroscopy (MS/MS). Rapid Commun. Mass Spectrom. 6, 528-530 (1992)

15. Lammert, S.A., Cooks, R.G.: Surface-induced dissociation of molecular-ions in a quadrupole ion trap mass-spectrometer. J. Am. Soc. Mass Spectrom. 2, 487-491 (1991)

16. Jullian, R.K., Nappi, M., Weil, C., Cooks, R.G.: Multiparticle simulation of ion motion in the ion trap mass spectrometer: resonant and direct current pulse excitation. J. Am. Soc. Mass Spectrom. 6, $57-$ 70 (1995)

17. Cooks, R.G., Cleven, C.D., Horn, L.A., Nappi, M., Weil, C., Soni, M. H., Julian, R.K.: Nondestructive detection of ions in a quadrupole ion trap using a DC pulse to force coherent ion motion: a simulation study. Int. J. Mass Spectrom. Ion Processes 146/147, 147-163 (1995)

18. Plass, W.R.: Theory of dipolar DC excitation and DC tomography in the rf quadrupole ion trap. Int J Mass Spectrom. 202, 175-197 (2000)
19. Tolmachev, A.V., Vilkov, A.N., Bogdanov, B., Păsa-Tolić, L., Masselon, C.D., Smith, R.D.: Collisional activation of ions in $\mathrm{rf}$ ion traps and ion guides: The effective ion temperature treatment. J. Am. Soc. Mass Spectrom. 15, 1616-1628 (2004)

20. Webb, I.K., Londry, F.A., McLuckey, S.A.: Implementation of dipolar DC CID in storage and transmission modes on a quadrupole/time-offlight tandem mass spectrometer. Rapid Commun. Mass Spectrom. 25, 2500-2510 (2011)

21. Prentice, B.M., Xu, W., Ouyang, Z., McLuckey, S.A.: DC potentials applied to an end-cap electrode of a 3-D ion trap for enhanced $M^{n}$ functionality. Int J Mass Spectrom. 306, 114-122 (2011)

22. Prentice, B.M., Santini, R.E., McLuckey, S.A.: Adaptation of a 3-D Quadrupole Ion Trap for Dipolar DC Collisional Activation. J. Am. Soc. Mass Spectrom. 22, 1486-1492 (2011)

23. Brodbelt, J.S.: Shedding light on the frontier of photodissociation. $J$. Am. Soc. Mass Spectrom. 22, 197-206 (2011)

24. Plass, W.R., Gill, L.A., Bui, H.A., Cooks, R.G.: Ion mobility measurement by DC tomography in an $\mathrm{rf}$ quadrupole ion trap. J. Phys. Chem. 104, 5059-5065 (2000)

25. Wells, J.M., Chrisman, P.A., McLuckey, S.A.: Dueling electrospray: instrumentation to study ion/ion reactions of electrospray-generated cations and anions. J. Am. Soc. Mass Spectrom. 13, 614-622 (2002)

26. Syka, J.E.P.: Commercialization of the quadrupole ion trap. Practical Aspects of Ion Trap Mass Spectrometry. Vol. I, CRC Press: Boca Raton, FL, 1995 (Chapter 4)

27. Sztáray, J., Memboeuf, A., Drahos, L., Vékey, K.: Leucine enkephalin - a mass spectrometry standard. J. Mass Spectrom. 30, 298-320 (2011)

28. Asano, K.G., Goeringer, D.E., McLuckey, S.A.: Thermal dissociation in the quadrupole ion trap: ions derived from leucine enkephalin. DOI:dx. doi.org Int. J. Mass Spectrom. Ion Processes 185/186/187, 207-219 (1999)

29. Goeringer, D.E., Asano, K.G., McLuckey, S.A.: Ion internal temperature and ion trap collisional activation: protonated leucine enkephalin. Int. J. Mass Spectrom. 182/183, 275-288 (1999)

30. Asano, K.G., Butcher, D.J., Goeringer, D.E., McLuckey, S.A.: Effective ion internal temperatures achieved via boundary activation in the quadrupole ion trap: protonated leucine enkephalin. J. Mass Spectrom. 34, 691-698 (1999)

31. Price, W.D., Williams, E.R.: Activation of peptide ions by blackbody radiation: factors that lead to dissociation kinetics in the rapid energy exchange limit. J. Phys. Chem. 101, 8844-8852 (1997)

32. Sannes-Lowery, K., Griffey, R.H., Kruppa, G.H., Speir, J.P., Hofstadler, S. A.: Multipole storage assisted dissociation, a novel in-source dissociation technique for electrospray ionization generated ions. Rapid Commun. Mass Spectrom. 12, 1957-1961 (1998)

33. Håkansson, K., Axelsson, J., Palmblad, M., Håkansson, P.: Mechanistic studies of multipole storage assisted dissociation. J. Am. Soc. Mass Spectrom. 11, 210-217 (2000) 